\title{
ASSISTÊNCIA DE AR NA DEPOSIÇÃO E PERDAS DE PRODUTOS FITOSSANITÁRIOS EM PULVERIZAÇÕES NA CULTURA DA SOJA
}

\author{
Fernando César Bauer ${ }^{1,3 *}$; Carlos Gilberto Raetano² \\ ${ }^{1}$ Pós-Graduando do Depto. de Defesa Fitossanitária - FCA/UNESP - Campus de Botucatu. \\ ${ }_{3}^{2}$ Depto. de Defesa Fitossanitária - FCA/UNESP - Campus de Botucatu, C.P. 237 - CEP: 18603-970 - Botucatu, SP. \\ ${ }^{3}$ Bolsista CAPES. \\ *Autor correspondente<fernandobauer@fca.unesp.br>
}

RESUMO: Com o objetivo de se comparar a deposição e as perdas em pulverizações na cultura da soja dois pulverizadores tratorizados de barra sendo um convencional (Condor M12-Jacto) e outro com assistência de ar junto a barra de pulverização (Columbia Vortex-Jacto) foram calibrados para aplicar $100 \mathrm{~L} \mathrm{ha}^{-1}$, respectivamente das soluções de $\mathrm{NaCl}$ e $\mathrm{KCl}$ a $2 \%(\mathrm{p} / \mathrm{v})$ à pressão de $207 \mathrm{kPa}\left(30 \mathrm{lbf} \mathrm{pol}^{-2}\right)$ e velocidade de $6 \mathrm{~km} \mathrm{~h}^{-1}$. Ambos os equipamentos utilizaram bicos de jato plano tipo API 110-1,5 espaçados a 0,5 $\mathrm{m}$ trabalhando a 0,4 $\mathrm{m}$ acima da cultura. A avaliação da deposição deu-se em folhas das partes superior, média e inferior das plantas e as perdas para o solo em alvos artificiais (placas de fórmica) distribuídos no interior da cultura de soja. Além desse alvo artificial outros tipos de coletores (limpadores de cachimbo) também foram utilizados em ambos os lados da cultura para se estimar a deriva. Os resultados mostraram que não houve diferença significativa na deposição entre os equipamentos em teste na parte superior das plantas de soja, mas houve diferença significativa nos depósitos obtidos nos terços médio e inferior, com vantagem para o equipamento com assistência de ar. A deriva foi consideravelmente maior para o equipamento convencional nas mesmas condições meteorológicas.

Palavras-chave: Glycine max, pulverizador tratorizado

\section{AIR-ASSISTED SPRAYER FOR THE EVALUATION OF DEPOSITION AND LOSSES OF PESTICIDE APPLICATION TO SOYBEAN CROP}

\begin{abstract}
The spray deposition and losses of pesticide application were evaluated for the soybean crop. In this study two sprayers were used: the air-assisted sprayer (Vortex-Jacto) and the conventional non-assisted sprayer (Condor M12-Jacto), both with API 110-1.5 flat fan nozzles operating at 30 psi working pressure, with $0.5 \mathrm{~m}$ spacing on the boom. Volume rates were $100 \mathrm{~L} \mathrm{ha}^{-1}$ for both equipments. A completely randomized experiment with four replicates was carried out in the field. For all treatments, the ground speed was $6 \mathrm{~km} \mathrm{~h}^{-1}$ and boom height was $0.40 \mathrm{~m}$ above canopy. Spray deposition and losses were evaluated using $2 \% \mathrm{KCl}$ and $\mathrm{NaCl}$ solutions in air-assisted and conventional non assisted sprayers. Deposition evaluations were performed on leaves from the upper, medium and lower parts of the soybean plant. Spray losses to the soil were evaluated using artificial targets (square wood board) that were distributed on the soil surface at intervals of $3 \mathrm{~m}$ from each other. Drift was evaluated through these targets and pipe cleaners fixed on rods placed at the left and right sides at the crop. There were no significant differences in the deposition for both equipments in the upper parts of the plants, but there were differences in the medium and lower parts, showing an advantage of the air-assisted sprayer. Drift and losses were larger for the conventional non assisted sprayer at similar meteorological conditions. Key words: Glycine max, mechanized sprayer
\end{abstract}

\section{INTRODUÇÃO}

A necessidade de melhoria na eficiência das aplicações de produtos fitossanitários tem sido relatada por vários pesquisadores como Adam (1977), Matuo (1990), Van De Zande et al. (1994) entre outros. Assim, o estudo e desenvolvimento de novas tecnologias de aplicação torna-se indispensável para a obtenção de melhores índices de eficiência de controle, que estão diretamente relacionados com a qualidade da aplicação. Da mesma forma Matthews (1992) afirma que os produtos fitossanitários devem ser aplicados com o máximo de eficiência e para isso torna-se necessário o estudo da deposição, cobertura e deriva, sendo esta última responsável por perdas, além de constituir num fator de contaminação ambiental.
Paschoal (1979) relata que partículas de defensivos em suspensão podem ser levadas pelo vento e contaminar alimentos e forragens em áreas não tratadas e, partículas menores, usualmente produzem contaminações mais sérias a maiores distâncias da área de aplicação. Os efeitos dos defensivos em áreas vizinhas àquelas que receberam a aplicação também foram estudados por Marshall (1987), concluindo que muitas espécies são severamente afetadas por contaminações acidentais ou deriva, principalmente de herbicidas; Sorthia \& Chari (1985) constataram 100\% de mortalidade em abelhas melíferas (Apis florea F. e Apis melipona L.) quando atingidas pela deriva de inseticidas fosforados.

A aplicação de defensivos com o uso da assistência de ar nos pulverizadores como forma auxiliar 
de melhoria da eficiência é comumente utilizada em aplicações em pomares, mas ainda pouco utilizada em lavouras anuais, segundo Furness (1991). No entanto, Hislop (1991) diz que muito embora a idéia de se usar a assistência de ar em pulverizações não seja nova, notase que, com a comercialização desses equipamentos, estudos mais detalhados devam ser efetuados em relação ao uso dessa tecnologia.

Cooke et al. (1990) comparando um pulverizador convencional com um equipamento dotado de assistência de ar junto a barra de pulverização demonstram que o equipamento com assistência de ar pode aumentar ou diminuir a deriva, se comparado ao equipamento convencional, dependendo da idade das plantas e do tipo de cultura alvo, da configuração operacional, inclusive a velocidade do ar na barra de pulverização, e do tipo de bico utilizado. Concluem que o uso da assistência de ar pode contribuir para a redução na dosagem dos agroquímicos, mas, para que isso ocorra muita pesquisa e desenvolvimento serão necessários para diminuir a deriva causada por esses equipamentos a níveis iguais ou menores que os convencionais.

Hoje é consenso a necessidade de se obter uma melhoria na eficiência da aplicação de produtos fitossanitários. Matuo (1990) e Matthews (1992) afirmam que essa melhoria deverá ser alcançada com a evolução no processo de aplicação em todos os seus aspectos, através do desenvolvimento de novos equipamentos capazes de cumprir essa tarefa com maior eficiência. Dessa forma, o estudo e desenvolvimento de novas tecnologias de aplicação se torna indispensável na medida em que se pretende aprimorar o processo.

Assim sendo, o presente trabalho teve como ou por objetivos a avaliação e comparação da deposição e deriva em pulverizações utilizando equipamentos tratorizados sem (convencional) e com assistência de ar na barra de pulverização, visando estimar as possíveis perdas durante a aplicação de produtos fitossanitários.

\section{MATERIAL E MÉTODOS}

Os pulverizadores sem assistência de ar convencional, Modelo Condor M-12 (Pco) e com assistência de ar junto a barra de pulverização Modelo Columbia Vortex, (Pca) ambos fabricados por Máquinas Agrícolas Jacto S.A., foram calibrados para aplicar $100 \mathrm{~L} \mathrm{ha}^{-1}$ de soluções de $\mathrm{NaCl}$ e $\mathrm{KCl}$ a $2 \%$ (massa / volume), respectivamente, ambos trabalhando a pressão de $207 \mathrm{KPa}\left(30 \mathrm{lbf} \mathrm{pol}^{-2}\right.$ ) e velocidade de deslocamento igual a $6 \mathrm{~km} \mathrm{~h}^{-1}$ e equipados com bicos jato plano API 1101,5 (Jacto) espaçados a $0,5 \mathrm{~m}$. No Pca a velocidade média do ar na barra de pulverização foi de $24,8 \mathrm{~km} \mathrm{~h}^{-1}$. Nesse equipamento trabalhou-se com barra de $18 \mathrm{~m}$ fechando-se os bicos situados nos $3 \mathrm{~m}$ finais de ambos os lados da barra que, com isso, ficou com $12 \mathrm{~m}$ de faixa efetiva de aplicação, comprimento igual àquele utilizado no Pco.
A aplicação dos sais foi feita em alvos naturais (cultura de soja no estádio R6 de desenvolvimento, segundo classificação de Fehr et al., citado por Müller, 1981) e artificiais (placas de fórmica de $0,10 \times 0,10 \mathrm{~m}$ ) com a barra de pulverização de ambos os equipamentos a $40 \mathrm{~cm}$ acima das plantas. A aplicação foi feita simultaneamente pelos equipamentos em uma única passagem, alternando uma vez a ordem de passagem durante a pulverização. Inicialmente o Pco se deslocou a frente seguido pelo Pca a $10 \mathrm{~m}$ de distância até a metade do percurso onde a ordem se inverteu com o Pco se deslocando $10 \mathrm{~m}$ atrás do Pca.

No momento da pulverização as condições ambientais foram: temperatura média $=27,9^{\circ} \mathrm{C}$; umidade relativa $=66,1 \%$; velocidade média do vento $=0,5 \mathrm{~m} / \mathrm{s}$.

Para a avaliação do volume de calda recuperado em alvo natural foram colhidos 6 folíolos (sendo 2 do terço superior, 2 do terço médio e 2 do terço inferior) de 7 plantas posicionadas lado a lado no sentido perpendicular ao deslocamento dos equipamentos, porém em linhas de plantio alternadas, em 4 pontos previamente marcados para amostragem ao longo do percurso do equipamento. Placas de fórmica $(0,10 \times 0,10 \mathrm{~m})$, em número de 5 , foram colocadas, internamente à cultura, perpendicular ao sentido do deslocamento do equipamento, a $10 \mathrm{~cm}$ de altura do solo e espaçadas entre si de forma aleatória para estimar o volume de calda recuperado e, dessa forma, quantificar as perdas da pulverização para o solo em ambos os equipamentos. Dez placas foram colocadas, sendo 5 entre as linhas de plantio, no interior da cultura e 5 fora da cultura. A disposição dos alvos naturais e artificiais (placas de fórmica) no local do experimento são apresentadas na Figura 1.

$\mathrm{Na}$ avaliação da deriva utilizou-se de alvos coletores artificiais sendo: a) placas de fórmica distanciadas de $3 \mathrm{~m}$ entre si afixadas a $10 \mathrm{~cm}$ de altura do solo,

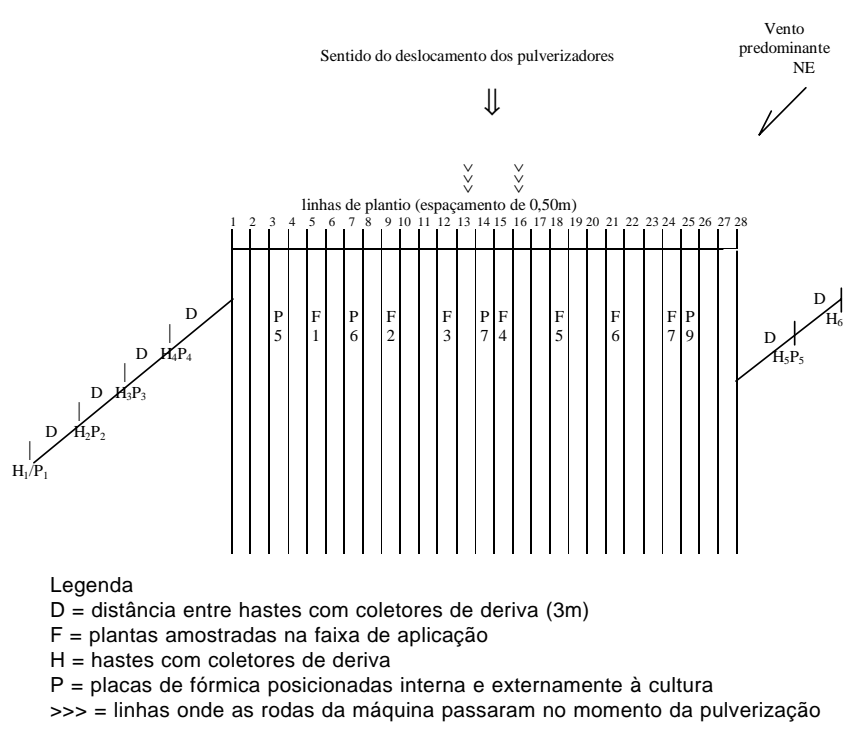

Figura 1 - Localização dos alvos em um ponto de amostragem na área experimental. 
externamente à cultura e b) limpadores de cachimbo (arames de aço recobertos com fios de algodão encontrados em qualquer tabacaria e utilizados na limpeza de cachimbos tendo comprimento e diâmetro médio de $16,5 \mathrm{~cm}$ e $0,3 \mathrm{~cm}$, respectivamente), fixados em hastes de bambú alinhadas na mesma direção e a $45^{\circ}$ em relação às linhas da cultura. Do lado esquerdo da cultura foram colocadas hastes a 3 e $6 \mathrm{~m}$ de distância da ponta da barra de pulverização e a 3, 6, 9 e $12 \mathrm{~m}$ do lado direito. A distribuição desigual das hastes de sustentação dos coletores deuse em função do sentido e direção predominante do vento na área experimental (Figura 1). Nas hastes distanciadas em $3 \mathrm{~m}$ de ambos os lados os coletores foram colocados a 0,$25 ; 0,50 ; 0,75 ; 1,00 ; 1,50$ e $2,00 \mathrm{~m}$ de altura do solo e nas demais $1,2,3,4,5$, e $6 \mathrm{~m}$ de altura.

Após a pulverização e secagem da calda aplicada todos os coletores foram retirados e armazenados em sacos de papel e estes acondicionados em sacos plásticos mantidos sob refrigeração. Após 12 horas os folíolos foram lavados em $20 \mathrm{ml}$ de água, os limpadores de cachimbo em $30 \mathrm{ml}$ e as placas de fórmica em $50 \mathrm{ml}$, sendo utilizada água deionizada para a lavagem de todas as amostras. Da solução resultante da lavagem dos coletores artificiais foi separada uma alíquota de $20 \mathrm{ml}$ colocadas em vidros de igual volume identificados e levados para quantificação através do espectrofotômetro de ab-sorção atômica (Marca Carl Zeiss-Jena, Modelo AAS-30).

A concentração inicial de $\mathrm{K}$ e $\mathrm{Na}$ na calda aplicada, obtida no mesmo espectrofotômetro foi de $7.596,67$ e 5.046,67 $\mathrm{mg} \mathrm{L}^{-1}$, respectivamente. Os níveis de depósitos para os sais foram avaliados em alvos naturais (superfície foliar) e artificiais (placas de fórmica) e ao correlacioná-los com a quantidade presente nas caldas de aplicação foi possível, através da equação 1 , estimar 0 volume líquido capturado $\left(\mu \mathrm{cm}^{-2}\right)$ por esses alvos em relação ao aplicado.

onde:

$$
C_{i} \cdot V_{i}=C_{f} \cdot V_{f}
$$

$\mathrm{C}_{\mathrm{i}}=$ concentração de $\mathrm{K}$ ou $\mathrm{Na}$ na calda $\left(\mathrm{mg} \mathrm{L}^{-1}\right)$

$\mathrm{C}_{\mathrm{f}}=$ concentração de $\mathrm{K}$ ou $\mathrm{Na}$ detectada no espectrofotômetro $\left(\mathrm{mg} \mathrm{L}^{-1}\right)$

$V_{i}=$ volume capturado pelo alvo (ml)

$\mathrm{V}_{\mathrm{f}}=$ volume da diluição da amostra $(\mathrm{ml})$
A estimativa da área foliar, necessária para o cálculo dos depósitos (Equação 1) foi feita através da coleta, antes da pulverização, de folíolos em diferentes pontos da cultura. A medição do comprimento e largura máximos e da área foliar obtida através da análise de imagem, possibilita o cálculo de um fator que, uma vez multiplicado pela resultante da multiplicação do comprimento e a largura, fornece a área foliar em $\mathrm{cm}^{2}$.

A dificuldade de se avaliar a área de exposição de cada limpador de cachimbo impossibilitou o cálculo do volume de calda capturado por unidade de área. Dessa forma optou-se por avaliar a deriva através do volume de calda capturado por cada coletor.

Os dados médios dos depósitos de $\mathrm{K}$ e $\mathrm{Na}$ detectados nos alvos naturais (folíolos) e artificiais (placas de fórmica) foram comparados pelo Teste T. Os dados médios de deriva ( $\mu$ l/coletor-limpador de cachimbo) foram analisados no delineamento inteiramente casualizado no esquema fatorial $2 \times 4 \times 6$, sendo 2 equipamentos, 4 distâncias e 6 alturas dos coletores em relação à cultura e no esquema fatorial $2 \times 2 \times 6$, sendo 2 equipamentos, 2 posições (lados direito e esquerdo) e 6 alturas dos coletores em relação à cultura e comparados pelo Teste T ao nível de $5 \%$ de probabilidade.

O espectro de gotas produzidas pelo bico API 110-1,5 operado a $207 \mathrm{KPa}\left(30 \mathrm{lbf} \mathrm{pol}^{-2}\right)$, caracterizado pelo analizador de gotas Mastersizer (fabricado por Malvern Instruments) pertencente ao Departamento de Defesa Fitossanitária da Faculdade de Ciências Agrárias e Veterinárias/UNESP - Campus de Jaboticabal, revelou um diâmetro mediano volumétrico (vmd) de $143 \mu \mathrm{m}$ e o diâmetro mediano numérico (nmd) de $1,59 \mu \mathrm{m}$.

\section{RESULTADOS E DISCUSSÃO}

Os resultados do volume de calda capturado em diferentes posições da planta são mostrados na TABELA 1. De modo geral, o tratamento com assistência de ar junto a barra de pulverização proporcionou maiores níveis de volume de calda capturado demonstrando, com isso, maiores depósitos de $\mathrm{K}$ em relação ao $\mathrm{Na}$. $\mathrm{A}$ análise do volume capturado em folíolos nas diferentes posições

TABELA 1 - Volume médio de calda capturado por unidade de área em diferentes posições da planta após pulverização dos sais $\mathrm{KCl}$ e $\mathrm{NaCl}$ por diferentes equipamentos. Botucatu, SP. 1998.

\begin{tabular}{lccccc}
\hline & & \multicolumn{3}{c}{ Posição dos folíolos } \\
\cline { 3 - 6 } Equipamento & Traçador (sal) & inferior & mediana & superior & Planta \\
\hline sem assistência de ar & $\mathrm{NaCl}$ & $0,026 \mathrm{~b}^{*} \mathrm{~A}^{* *}$ & $0,025 \mathrm{bA}$ & $0,033 \mathrm{aA}$ & $0,028 \mathrm{~b}$ \\
com assistência de ar & $\mathrm{KCl}$ & $0,041 \mathrm{aA}$ & $0,037 \mathrm{aAB}$ & $0,030 \mathrm{aB}$ & $0,036 \mathrm{a}$ \\
\hline CV $(\%)$ & - & 63,57 & 52,52 & 56,50 & 58,71 \\
\hline DMS & - & 0,0120 & 0,0091 & 0,0097 & 0,0059 \\
\hline
\end{tabular}

(*) - Médias, nas colunas, seguidas de mesma letra minúscula, não diferem estatisticamente pelo Teste T, a $5 \%$.

$\left({ }^{* *}\right)$ - Médias, na linha, seguidas pela mesma letra maiúscula não diferem estatisticamente pelo Teste $\mathrm{T}$ a $5 \%$. 
da planta revelou maiores níveis de depósitos nas posições inferior e média para o equipamento com assistência de ar. Entretanto, na parte superior não foram observadas diferenças significativas no nível de depósito da pulverização proveniente dos diferentes equipamentos.

O fato de não ter sido detectada diferença nos depósitos na parte superior das plantas revela que a assistência de ar, nas condições desse experimento, não foi suficiente para melhorar a deposição em relação ao equipamento convencional.

O efeito da assistência de ar proporcionou significativo aumento dos níveis de depósitos nos folíolos posicionados na parte inferior da planta, nesse caso, quase duas vezes maior. As prováveis razões para a ocorrência desse fato são o aumento da velocidade das gotas e a maior movimentação das folhas nessas posições o que poderia influenciar positivamente na captura das gotas pelas folhas, proporcionando maiores níveis de depósitos.

Com relação às perdas para o solo, com os coletores (placas de fórmica) posicionados internamente à cultura, não se constatou diferenças significativas no volume de calda capturado entre os tratamentos em teste (TABELA 2). No entanto, é interessante observar que os valores referentes às perdas para o solo foram altos para ambos os equipamentos.

Por outro lado, externamente à cultura o Pco proporcionou a obtenção de níveis de depósitos significativamente maiores. A grande diferença, entre os valores do volume de calda capturado por esses alvos, se deve, provavelmente, ao fato de as gotas ficarem em suspensão acima da cultura e, por isso, suscetíveis a mudança de trajetória pelo vento.

A análise, de forma geral, dos resultados da influência do equipamento de aplicação, distância e altura dos coletores na deriva, após a pulverização, revelou que estes fatores atuam de forma independente na deriva (TABELA 3). Entretanto, na presente análise pode-se, também, observar a existência de efeito do equipamento e de interações estatisticamente significativas entre os fatores equipamento e distância.
TABELA 3 - Influência do equipamento de aplicação, distância e altura dos coletores na deriva após pulverização de $\mathrm{KCl}$ e $\mathrm{NaCl}$ na cultura da soja.

\begin{tabular}{lrc}
\hline Fatores de variação & GL & $\begin{array}{c}\text { Valores de F e médias } \\
\text { de deriva }(\mu \text { l por coletor) }\end{array}$ \\
\hline Equipamento (E) & 1 & $2.019,65^{*}$ \\
Distância (D) & 3 & 1,14 n.s. \\
Altura (H) & 5 & 0,17 n.s. \\
E x D & 3 & $3,38^{*}$ \\
E x H & 5 & 0,29 n.s. \\
D x H & 15 & 0,38 n.s. \\
E x D x H & 15 & 0,45 n.s. \\
\hline CV $(\%)$ & & 25,13 \\
\hline
\end{tabular}

Médias da deriva ( $\mu$ l por coletor)

\begin{tabular}{ll}
\hline Equipamento com & $0,489 \mathrm{~A}$ \\
assistência de $\operatorname{ar}\left(E_{1}\right)$ & $4,809 \mathrm{~B}$ \\
$\begin{array}{l}\text { Equipamento sem } \\
\text { assistência de } \operatorname{ar}\left(\mathrm{E}_{2}\right)\end{array}$ & 0,269 \\
\hline DMS 5\% & $2,683 \mathrm{~A}$ \\
\hline $6 \mathrm{~m} \mathrm{LD}\left(\mathrm{D}_{1}\right)$ & $2,687 \mathrm{~A}$ \\
$9 \mathrm{~m} \mathrm{LD}\left(\mathrm{D}_{2}\right)$ & $2,728 \mathrm{~A}$ \\
$12 \mathrm{~m} \mathrm{LD}\left(\mathrm{D}_{3}\right)$ & $2,498 \mathrm{~A}$ \\
\hline $6 \mathrm{~m} \mathrm{LE}\left(\mathrm{D}_{1}\right)$ & 0,269 \\
\hline $\mathrm{DMS} \mathrm{5} \%$ & $2,708 \mathrm{~A}$ \\
\hline $1 \mathrm{~m}\left(\mathrm{H}_{1}\right)$ & $2,655 \mathrm{~A}$ \\
$2 \mathrm{~m}\left(\mathrm{H}_{2}\right)$ & $2,575 \mathrm{~A}$ \\
$3 \mathrm{~m}\left(\mathrm{H}_{3}\right)$ & $2,641 \mathrm{~A}$ \\
$4 \mathrm{~m}\left(\mathrm{H}_{4}\right)$ & $2,693 \mathrm{~A}$ \\
$5 \mathrm{~m}\left(\mathrm{H}_{5}\right)$ & $2,622 \mathrm{~A}$ \\
\hline $6 \mathrm{~m}\left(\mathrm{H}_{6}\right)$ & 0,329 \\
\hline $\mathrm{DMS} 5 \%$ & \\
\hline
\end{tabular}

* = significativo a $5 \%$.

Médias na mesma coluna e dentro do mesmo fator não diferem estatisticamente a $5 \%$ pelo Teste $\mathrm{T}$.

${ }^{1} \mathrm{LD}=$ Lado direito; ${ }^{2} \mathrm{LE}=$ Lado esquerdo.

TABELA 2 - Volume médio de calda capturado por unidade de área $\left(\mu \mathrm{l} \mathrm{cm}^{-2}\right)$ e porcentagem em relação ao total aplicado em alvos artificiais (placas de fórmica) posicionados interna e externamente à cultura de soja após pulverização de sais $\mathrm{KCl}$ e $\mathrm{NaCl}$ por diferentes equipamentos. Botucatu, SP. 1998.

\begin{tabular}{|c|c|c|c|c|c|}
\hline \multirow{3}{*}{ Equipamento } & \multirow{3}{*}{$\begin{array}{c}\text { Traçador } \\
\text { (sal) }\end{array}$} & \multicolumn{4}{|c|}{ Posição do alvo em relação à cultura } \\
\hline & & \multicolumn{2}{|c|}{ interna } & \multicolumn{2}{|c|}{ externa } \\
\hline & & $\mu \mathrm{l} \mathrm{cm^{-2 }}$ & $\%$ & $\mu \mathrm{lcm} \mathrm{cm}^{-2}$ & $\%$ \\
\hline sem assistência de ar & $\mathrm{NaCl}$ & $0,203 \mathrm{a}$ & 18,97 & $0,050 \mathrm{a}$ & 4,67 \\
\hline com assistência de ar & $\mathrm{KCl}$ & $0,152 \mathrm{a}$ & 15,05 & $0,008 \mathrm{~b}$ & 0,79 \\
\hline CV (\%) & - & \multicolumn{2}{|c|}{81,79} & \multicolumn{2}{|c|}{93,73} \\
\hline DMS & - & \multicolumn{2}{|c|}{0,093} & \multicolumn{2}{|c|}{0,017} \\
\hline
\end{tabular}

Médias seguidas de mesma letra, na mesma coluna, não diferem estatisticamente pelo Teste T, a 5\%. 
Níveis de deriva significativamente maiores, da ordem de 9 vezes, foram detectados na aplicação com o Pco em relação ao Pca (TABELA 3).

Para a interação equipamento $x$ distância podese constatar o efeito do fator equipamento dentro de cada nível do fator distância. O fator distância dentro de equipamento somente mostrou efeito quando os coletores estavam posicionados do lado esquerdo a $6 \mathrm{~m}$ de distância da cultura, para o equipamento convencional (TABELA 4).

Da observação da TABELA 4 pode-se constatar níveis significativamente menores de deriva para o Pca em todas as distâncias dos coletores em teste.

Níveis significativamente menores de deriva com o Pco foram detectados nos coletores posicionados a $6 \mathrm{~m}$ de distância do lado esquerdo da cultura comparativamente às demais distâncias em teste. Isso provavelmente ocorreu devido ao posicionamento desses coletores em relação ao vento predominante no momento da aplicação (Figura 1).

Os maiores níveis de deriva do Pco se deve, provavelmente, ao maior tempo de permanência acima da cultura das gotas pulverizadas fazendo com que essas gotas ficassem suscetíveis às condições ambientais. Por outro lado, a menor deriva com o Pca se deve, provavelmente, a não permanência acima da cultura de parte das gotas pulverizadas que, sob influência da assistência de ar, se deslocaram para o interior da cultura.

De modo geral a análise da influência dos fatores equipamento de aplicação, posição e altura dos coletores distanciados a $3 \mathrm{~m}$ de ambos os lados da cultura também mostrou que esses fatores agem de forma independente porém, os fatores equipamento e posição do coletor (lados direito e esquerdo) apresentaram efeito significativo na deriva (TABELA 5).

Para a interação equipamento $x$ posição do coletor, quando posicionados a $3 \mathrm{~m}$ da cultura, a análise da deriva revelou a existência de efeito do fator equipamento dentro de cada nível do fator posição de coletor, no entanto não se observou efeito do fator posição do coletor dentro do fator equipamento (TABELA 6).

TABELA 4 - Médias de deriva ( $\mu \mathrm{l})$ após a pulverização dos sais $\mathrm{KCl}$ e NaCl na cultura da soja para a interação Equipamento x Distância

\begin{tabular}{lrrrr}
\hline Equipamento & \multicolumn{4}{c}{ Distância (metros) } \\
\cline { 2 - 5 } & $6 \mathrm{LD}^{1}$ & $9 \mathrm{LD}$ & $12 \mathrm{LD}$ & $6 \mathrm{LE}^{2}$ \\
\hline $\begin{array}{l}\text { Com assistên- } \\
\text { cia de ar }\left(\mathrm{E}_{1}\right)\end{array}$ & $0,442 \mathrm{a}^{*} / \mathrm{A}^{* *}$ & $0,390 \mathrm{a} / \mathrm{A}$ & $0,544 \mathrm{a} / \mathrm{A}$ & $0,581 \mathrm{a} / \mathrm{A}$ \\
$\begin{array}{l}\text { Convencional } \\
\left(\mathrm{E}_{2}\right)\end{array}$ & $5,013 \mathrm{~b} / \mathrm{A}$ & $4,983 \mathrm{~b} / \mathrm{A}$ & $4,822 \mathrm{~b} / \mathrm{A}$ & $4,416 \mathrm{~b} / \mathrm{B}$ \\
\hline
\end{tabular}

$1 \mathrm{LD}=$ Lado direito: 2 LE = Lado Esquerdo

* Médias, na coluna, seguida de mesma letra minúscula não diferem pelo Teste $\mathrm{T}$ a $5 \%$.

**Médias, na linha, seguidas de mesma letra maiúscula não diferem pelo Teste $\mathrm{T}$ a $5 \%$.
TABELA 5 - Influência do equipamento de aplicação, posição e altura dos coletores na deriva após a pulverização de $\mathrm{KCl}$ e NaCl distanciadas à 3 metros da cultura da soja.

\begin{tabular}{|c|c|c|}
\hline Fatores de variação & GL & $\begin{array}{l}\text { Valores de } \mathrm{F} \text { e médias } \\
\text { de deriva }(\mu \mathrm{l} \text { por coletor })\end{array}$ \\
\hline Equipamento (E) & 1 & $665,64^{*}$ \\
\hline Posição do coletor $(P)$ & 1 & $3,74^{*}$ \\
\hline Altura do coletor $(\mathrm{H})$ & 5 & 0,53 n.s. \\
\hline$E \times P$ & 1 & 0,66 n.s. \\
\hline $\mathrm{E} \times \mathrm{H}$ & 5 & 0,17 n.s. \\
\hline $\mathrm{P} \times \mathrm{H}$ & 5 & 0,29 n.s. \\
\hline $\mathrm{E} \times \mathrm{P} \times \mathrm{H}$ & 5 & 0,06 n.s. \\
\hline CV (\%) & & 28,17 \\
\hline
\end{tabular}

Médias da deriva ( $\mu$ l por coletor)

Equipamento com assistência de $\operatorname{ar}\left(E_{1}\right)$

$0,667 \mathrm{~B}$

Equipamento sem assistência de $\operatorname{ar}\left(E_{2}\right)$

$4,502 \mathrm{~A}$

\begin{tabular}{ll}
\hline DMS 5\% & 0,054 \\
\hline Lado direito $\left(\mathrm{P}_{1}\right)$ & $2,728 \mathrm{~A}$ \\
Lado e squerdo $\left(\mathrm{P}_{2}\right)$ & $2,441 \mathrm{~A}$ \\
\hline DMS 5\% & 0,296 \\
\hline $0,25 \mathrm{~m}\left(\mathrm{H}_{1}\right)$ & $2,439 \mathrm{~A}$ \\
$0,50 \mathrm{~m}\left(\mathrm{H}_{2}\right)$ & $2,492 \mathrm{~A}$ \\
$0,75 \mathrm{~m}\left(\mathrm{H}_{3}\right)$ & $2,701 \mathrm{~A}$ \\
$1,00\left(\mathrm{H}_{4}\right)$ & $2,786 \mathrm{~A}$ \\
$1,50 \mathrm{~m}\left(\mathrm{H}_{5}\right)$ & $2,560 \mathrm{~A}$ \\
$2,00 \mathrm{~m}\left(\mathrm{H}_{6}\right)$ & $2,529 \mathrm{~A}$ \\
\hline DMS 5\% & 0,512 \\
\hline
\end{tabular}

* significativo a $5 \%$.

Médias na mesma coluna e dentro do mesmo fator não diferem estatisticamente a $5 \%$ pelo Teste $\mathrm{T}$.

TABELA 6 - Médias de deriva $(\mu \mathrm{l})$ após a pulverização dos sais $\mathrm{KCl}$ e $\mathrm{NaCl}$ na cultura da soja para a interação Equi pamento x Posição do Coletor.

\begin{tabular}{lcc}
\hline Equipamento & \multicolumn{3}{c}{ Posição a 3 metros da cultura } \\
\cline { 2 - 3 } & lado direito & lado esquerdo \\
\hline com assistência de $\operatorname{ar}\left(E_{1}\right)$ & $0,750 \mathrm{~b}^{*} / \mathrm{A}^{\star *}$ & $0,584 \mathrm{~b} / \mathrm{A}$ \\
sem assistência de $\operatorname{ar}\left(\mathrm{E}_{2}\right)$ & $4,706 \mathrm{a} / \mathrm{A}$ & $4,297 \mathrm{a} / \mathrm{A}$ \\
\hline
\end{tabular}

* Médias, na coluna, seguidas pela mesma letra minúscula não diferem pelo Teste T a 5\%.

** Médias, na linha, seguidas pela mesma letra maiúscula não diferem pelo Teste $\mathrm{T}$ a $5 \%$.

Níveis médios significativamente maiores de deriva foram obtidos com o pulverizador convencional em relação ao pulverizador com assistência de ar junto a barra de pulverização, independente da posição dos coletores em relação a cultura (lados esquerdo e direito). 


\section{CONCLUSÕES}

- A assistência de ar junto a barra de pulverização promoveu significativo aumento da deposição nas porções média e inferior das plantas de soja.

- Redução significativa da deriva foi constatada com o uso da assistência de ar junto a barra de pulverização em relação ao equipamento sem assistência de ar, em mesmas condições meteorológicas.

- As perdas para o solo, na cultura da soja, não foram estatisticamente maiores para o equipamento convencional quando comparada com o equipamento provido de assistência de ar junto a barra de pulverização.

\section{REFERÊNCIAS BIBLIOGRÁFICAS}

ADAM, A.V. Importance of pesticide application equipment and related field practices in developing countries. In: WATSON, D.L.; BROWN, A.W. (Ed.) Pesticide management and insect resistance. New York: Academic Press, 1977. p.217-225.

COOKE, B.K.; HISLOP, E.C.; HERRINGTON, P.J.; WESTERN, N.M.; HUMPHERSON-JONES, F. Air-assisted spraying of arable crops, in relation to deposition, drift and pesticide performance. Crop Protection, v.9, p.303-311, 1990.

FURNESS, G.O. A comparison of simple bluff plate and axial fans for air-assisted, high-speed, low-volume spray application to wheat and sunflower plants. Journal of Agricultural Engineering Research, v.48, p.57-75, 1991.
HISLOP, E.C. Air assisted crop spraying: an introductory review. In: LAVERS, A.; HERINGTON, P.; SOUTHCOMBE, E.S.E. (Ed.) Air-assisted spraying in crop protection. Farnham: British Crop Protection Council, 1991. p.3-14. (Monograph, 46).

MARSHALL, E.J.P. Herbicide effects on the flora of arable field boundaries. In: BRITISH CROP PROTECTION CONFERENCE - WEEDS, Brighton, 1987. Proceedings. Brighton: BCPC, 1987. v.1, p.291-298.

MATTHEWS, G.A. Pesticide application methods. 2. ed. London: Longman, 1992. 405p.

MATUO, T. Tecnologia de aplicação de defensivos agrícolas. Jaboticabal: FUNEP, 1990. 139p.

MÜLLER, L. Morfologia, anatomia e desenvolvimento. In: MIYASAKA, S.; MEDINA, J.C. (Ed.) A soja no Brasil. Campinas: ITAL, 1981. 1062p.

PASCHOAL, A.D. Pragas, praguicidas e a crise ambiental: problemas e soluções. Rio de Janeiro: Fundação Getulio Vargas, 1979. 102p.

SORTHIA, B.K.; CHARI, M.S. Toxicity of some insecticides to honey bees, Apis florea F. and Apis melipona L. Journal of Entomological Research, v.9, p.195-197, 1985.

VAN DE ZANDE, J.C., MEIER, R., VAN IJZENDOORN, M.T. Airassisted spraying in winter wheat-results of deposition measurements and the biological effect of fungicides against leaf and ear diseases. In: BRITISH CROP PROTECTION CONFERENCE - PESTS AND DISEASES, Brighton, 1994. Proceedings. Brighton: BCPC, 1994. p.313-318.

Recebido em 25.06.99 